\title{
Spatial data compression and denoising via wavelet transformation
}

\begin{abstract}
A new interpolation wavelet filter for TIN data compression has been applied in two steps, namely splitting and lifting. In the splitting step, a triangle has been divided into several subtriangles and the elevation step has been used to 'modify' the point values (point coordinates for geometry) after the splitting. This data set is then compressed at the desired locations by using second-generation wavelets: scalar wavelets constructed by using a lifting scheme. Application of the compressed data compares favourably with results derived using the original (and much larger) TIN data set.
\end{abstract}

Keyword: Compression; Data set; Elevation; Interpolation; Spatial data; Triangulation; Wavelet 